S. Graf, Fakultät für Matematik und Informatik, Universität Passau, 94030 Passau, Germany. e-mail: graf@fmi.uni-passau.de

H. Luschgy, Fachbereich Mathematik IV, Universität Trier, 54286 Trier, Germany. e-mail: luschgy@uni-trier.de

\title{
ASYMPTOTICS OF THE QUANTIZATION ERRORS FOR SELF-SIMILAR PROBABILITIES
}

\begin{abstract}
The formulae for determining the quantization dimensions of selfsimilar probabilities satisfying the open set condition are proved by a new method. In addition, this method gives the exact order of convergence for the quantization errors.
\end{abstract}

\section{Introduction}

Given a Borel probability $P$ on $\mathbb{R}^{d}$, a number $r \in[0,+\infty]$ and a natural number $n \in \mathbb{N}$ the $n$-th quantization error of order $r$ for $P$ is defined by

$$
e_{n, r}= \begin{cases}\inf \left\{\exp \int \log d(x, \alpha) d P(x) \mid \alpha \subset \mathbb{R}^{d}, \operatorname{card}(\alpha) \leq n\right\} & \text { if } r=0 \\ \inf \left\{\left(\int d(x, \alpha)^{r} d P(x)\right)^{1 / r} \mid \alpha \subset \mathbb{R}^{d}, \operatorname{card}(\alpha) \leq n\right\} & \text { if } 0<r<\infty \\ \inf \left\{\sup _{x \in \operatorname{supp}(P)} d(x, \alpha) \mid \alpha \subset \mathbb{R}^{d}, \operatorname{card}(\alpha) \leq n\right\} & \text { if } r=\infty\end{cases}
$$

where $d(x, \alpha)$ denotes the distance of the point $x$ to the set $\alpha$ with respect to a given norm \|\| on $\mathbb{R}^{d}$. (One has to impose certain conditions on $P$ to guarantee that the integrals and the supremum in the above expressions exist in $\mathbb{R}$.) The quantization dimension of order $r$ for $P$ is

$$
D_{r}(P)=\lim _{n \rightarrow \infty} \frac{\log n}{-\log e_{n, r}}
$$

if this limit exists. For self-similar probabilities $P$ satisfying the open set condition and $0<r<\infty$ it was shown in [6] that the quantization dimension

Key Words: self-similar probability, quantization

Mathematical Reviews subject classification: 28A80 (primary) and 60E99 (secondary)

Received by the editors October 27, 2000 
$D_{r}(P)$ exists in $(0,+\infty)$ and a formula for its computation was derived. In the present note we give a new proof of these results and extend them to the cases $r=0$ and $r=+\infty$. Moreover we will show that, for all $r \in[0,+\infty]$, $0<\liminf _{n \rightarrow \infty} n e_{n, r}^{D_{r}} \leq \limsup _{n \rightarrow \infty} n e_{n}^{D_{r}}<+\infty$.

\section{Basic Notation and Definitions}

In what follows $N$ is always a natural number $\geq 2$ and $S_{1}, \ldots, S_{N}$ are contractive similitudes from $\mathbb{R}^{d}$ into itself. Let $s_{i}$ be the contraction number of $S_{i}$; i.e., $s_{i} \in(0,1)$ and $\left\|S_{i} x-S_{i} y\right\|=s_{i}\|x-y\|$ for all $x, y \in \mathbb{R}^{d}$. Sometimes the $N$-tuple $\left(S_{1}, \ldots, S_{N}\right)$ is called an iterated function system (IFS). Its attractor is the unique non-empty compact set $A$ in $\mathbb{R}^{d}$ with

$$
A=S_{1}(A) \cup \ldots \cup S_{N}(A) .
$$

For every probability vector $p=\left(p_{1}, \ldots, p_{N}\right)$ there exists a unique Borel probability $P$ on $\mathbb{R}^{d}$ which satisfies the equation $P=\sum_{i=1}^{N} p_{i} P \circ S_{i}^{-1} . P$ is called the self-similar probability corresponding to $\left(S_{1}, \ldots, S_{N ;} p\right)$. If each component $p_{i}$ of $p$ is strictly positive, then the support of $P$ equals $A$.

The IFS $\left(S_{1}, \ldots, S_{N}\right)$ is said to satisfy the open set condition (OSC) iff there is a non-empty open set $U$ in $\mathbb{R}^{d}$ with $S_{i}(U) \subset U$ and $S_{i}(U) \cap S_{j}(U)=\emptyset$ for all $i, j$ with $i \neq j$. According to a result of Schief [7] $U$ can be chosen to be bounded and such that $U \cap A \neq \emptyset$.

Let $\{1, \ldots, N\}^{*}$ be the set of finite words over the alphabet $\{1, \ldots, N\}$ including the empty word $\emptyset$. For $\sigma \in\{1, \ldots, N\}^{*}$ the length of $\sigma$ is denoted by $|\sigma|$. For $n \in \mathbb{N},\{1, \ldots, N\}^{n}$ is the set of all words of length $n$. A word $\sigma=\sigma_{1} \ldots \sigma_{n}$ is said to be a predecessor of a word $\tau=\tau_{1} \ldots \tau_{m}$, in symbols $\sigma \prec \tau$, iff $n \leq m$ an $\sigma_{i}=\tau_{i}$ for $i=1, \ldots, n$. The empty word is the predecessor of every word. Words $\sigma$ and $\tau$ are called incomparable if neither $\sigma \prec \tau$ nor $\tau \prec \sigma$. For $\sigma \in\{1, \ldots N\}^{*}$ set

$$
\begin{aligned}
& S_{\sigma}= \begin{cases}i d_{\mathbb{R}^{d}} & \text { if } \sigma=\emptyset \\
S_{\sigma_{1}} \circ \ldots \circ S_{\sigma_{n}} & \text { if } \sigma=\sigma_{1} \ldots \sigma_{n},\end{cases} \\
& A_{\sigma}=S_{\sigma}(A), \\
& s_{\sigma}= \begin{cases}1 & \text { if } \sigma=\emptyset \\
s_{\sigma_{1}} \cdot \ldots \cdot s_{\sigma_{n}} & \text { if } \sigma=\sigma_{1} \ldots \sigma_{n},\end{cases}
\end{aligned}
$$


and

$$
p_{\sigma}= \begin{cases}1 & \text { if } \sigma=\emptyset \\ p_{\sigma_{1}} \cdot \ldots \cdot p_{\sigma_{n}} & \text { if } \sigma=\sigma_{1} \ldots \sigma_{n} .\end{cases}
$$

If $\left(S_{1}, \ldots, S_{N}\right)$ satisfies the OSC, then $P\left(A_{\sigma} \cap A_{\tau}\right)=0$ if $\sigma$ and $\tau$ are incomparable and, moreover, $P\left(A_{\sigma}\right)=p_{\sigma}$ (see [2], Lemma 3.3).

\section{Statement of the Main Result}

Let $D_{\infty}$ be the unique real number with $\sum_{i=1}^{N} s_{i}^{D_{\infty}}=1$. Then $D_{\infty}$ is called the similarity dimension of $\left(S_{1}, \ldots, S_{N}\right)$. For $r \in(0,+\infty)$ there exists a unique $D_{r} \in(0,+\infty)$ satisfying $\sum_{i=1}^{N}\left(p_{i} s_{i}^{r}\right)^{\frac{D_{r}}{r+D_{r}}}=1$. (see [5], Lemma 14.4). Let

$$
D_{0}=\frac{\sum_{i=1}^{N} p_{i} \log p_{i}}{\sum_{i=1}^{N} p_{i} \log s_{i}}
$$

where $(0 \log 0:=0)$.

Theorem 3.1. Let $\left(S_{1}, \ldots, S_{N}\right)$ have the OSC, $p=\left(p_{1}, \ldots, p_{N}\right)$ with $p_{i}>0$ for all $i$, and let $P$ be the self-similar probability corresponding to $\left(S_{1}, \ldots, S_{N} ; p\right)$. Let $D_{r}$ be as above. Then, for every $r \in[0,+\infty]$,

$$
0<\liminf _{n \rightarrow \infty} n e_{n, r}^{D_{r}} \leq \limsup _{n \rightarrow \infty} n e_{n, r}^{D_{r}}<+\infty ;
$$

in particular $\lim _{n \rightarrow \infty} \frac{\log n}{-\log e_{n, r}}=D_{r}$.

\section{Remark 3.2.}

a) If $p=\left(s_{1}^{D_{\infty}}, \ldots, s_{N}^{D_{\infty}}\right)$, then $D_{r}=D_{\infty}$ for all $r \in[0,+\infty]$.

b) If $p \neq\left(s_{1}^{D_{\infty}}, \ldots, s_{N}^{D_{\infty}}\right)$, then the function $[0,+\infty] \rightarrow(0,+\infty), r \rightarrow D_{r}$ is strictly increasing and continuous.

Proof. That $(0,+\infty] \rightarrow(0,+\infty), r \rightarrow D_{r}$ is strictly increasing and continuous follows from Lemma 14.16 and the proof of Theorem 14.15 in [5]. (Actually the results there are stated for $r \geq 1$ only but the proofs work unchanged for 
$r>0$.) It remains to show that $\lim _{r \downarrow 0} D_{r}=D_{0}$. Let $F: \mathbb{R} \times \mathbb{R} \rightarrow \mathbb{R}$ be defined by $F(q, t)=\sum_{i=1}^{N} p_{i}^{q} s_{i}^{t}-1$. Then for every $q \in \mathbb{R}$ there exists a unique $\beta(q) \in \mathbb{R}$ with $F(q, \beta(q))=0$. By implicit differentiation the function $\mathbb{R} \rightarrow \mathbb{R}, q \rightarrow \beta(q)$ is differentiable with derivative

$$
\beta^{\prime}(q)=-\frac{\sum_{i=1}^{N} p_{i}^{q} s_{i}^{\beta(q)} \log p_{i}}{\sum_{i=1}^{N} p_{i}^{q} s_{i}^{\beta(q)} \log s_{i}} .
$$

Also, $\beta$ is strictly decreasing with $\lim _{q \rightarrow-\infty} \beta(q)=+\infty$ and $\lim _{q \rightarrow+\infty} \beta(q)=-\infty$ (see for instance, Falconer [1], p. 193). From the definitions we deduce that for $0<r<+\infty, \beta\left(\frac{D_{r}}{r+D_{r}}\right)=r \frac{D_{r}}{r+D_{r}}$. Since $\beta(1)=0$, we get

$$
\frac{\beta\left(\frac{D_{r}}{r+D_{r}}\right)-\beta(1)}{\frac{D_{r}}{r+D_{r}}-1}=\frac{r \frac{D_{r}}{r+D_{r}}}{-\frac{r}{r+D_{r}}}=-D_{r} .
$$

Thus $\lim _{r \downarrow 0} D_{r}=-\beta^{\prime}(1)=\frac{\sum_{i=1}^{N} p_{i} \log p_{i}}{\sum_{i=1}^{N} p_{i} \log s_{i}}=D_{0}$ if we can show that $\lim _{r \downarrow 0} \frac{D_{r}}{r+D_{r}}=1$. Since $0<\frac{D_{r}}{r+D_{r}}<1$ for all $r \in(0,+\infty)$ the claim is proved provided that, for every $r_{n} \downarrow 0$ for which $\left(\frac{D_{r_{n}}}{r_{n}+D_{r_{n}}}\right)_{n \in \mathbb{N}}$ converges to some $a$, it follows that $a=1$. But this obviously holds because $1=\lim _{n \rightarrow \infty} \sum_{i=1}^{N}\left(p_{i} s_{i}^{r_{n}}\right)^{\frac{D_{r_{n}}}{r_{n}+D_{r_{n}}}}=\sum_{i=1}^{N} p_{i}^{a}$.

c) It is an interesting question under what conditions the limit $\lim _{n \rightarrow \infty} n e_{n, r}^{D_{r}}$ exists. If $S_{1} \ldots S_{4}: \mathbb{R}^{2} \rightarrow \mathbb{R}^{2}$ are defined by $S_{i} x=\frac{1}{2} x+x_{i}$ with $x_{1}=(0,0)$, $x_{2}=\left(\frac{1}{2}, 0\right), x_{3}=\left(0, \frac{1}{2}\right), x_{4}=\left(\frac{1}{2}, \frac{1}{2}\right)$, and $p=\left(\frac{1}{4}, \frac{1}{4}, \frac{1}{4}, \frac{1}{4}\right)$, then the corresponding self-similar probability $P$ is the uniform distribution on the square $[0,1]^{2}$. In this case $D_{r}=2$ and $\lim _{n \rightarrow \infty} n e_{n, r}^{D_{r}}$ exists for all $r \in[0,+\infty]$ (see [5], Theorem 6.2 and Theorem 10.7 and [4], Theorem 3.2). If $S_{1}, S_{2}: \mathbb{R} \rightarrow \mathbb{R}$ are defined by $S_{1} x=\frac{1}{3} x$ and $S_{2} x=\frac{1}{3} x+\frac{2}{3}$ and $p=\left(\frac{1}{2}, \frac{1}{2}\right)$, then the corresponding self-similar probability $P$ is the uniform distribution on the classical Cantor set, the quantization dimension of order 2 is $D_{2}=\frac{\log 2}{\log 3}$, and the sequence $\left(n e_{n, 2}^{D_{2}}\right)_{n \in \mathbb{N}}$ does not converge (see [3], Theorem 6.3).

d) For general relationships between Hausdorff and box dimension of a probability $P$ and the quantization dimensions of $P$ the reader is referred to [4] 
and [5]. There he will also find a definition of upper and lower quantization dimensions together with their basic properties.

\section{Proof of the Main Result}

In this section we always assume that the assumptions of Theorem 3.1 are satisfied. Moreover, let $U$ be a bounded open subset of $\mathbb{R}^{d}$ with $A \cap U \neq \emptyset$, $S_{i}(U) \subset U$, and $S_{i}(U) \cap S_{j}(U)=\emptyset$ for $i \neq j$. That $\lim \sup n e_{n, r}^{D_{r}}<+\infty$ is shown in [5], Proposition 14.5 and 14.6 for $r \in(0,+\infty]$ and in [4], Theorem 5.3 for $r=0$. (Strictly speaking [5] only deals with $r \in[1,+\infty]$ but the results extend to $r \in(0,1)$ without change of proof). Here the OSC need not be assumed. Proposition 14.13 in [5] shows that $0<\liminf _{n \rightarrow \infty} n e_{n, \infty}^{D_{\infty}}$ and relies on the open set condition. That $0<\liminf _{n \rightarrow \infty} n e_{n, r}^{D_{r}} \leq \limsup _{n \rightarrow \infty} n e_{n, r}^{D_{r}}<\infty$ implies $\lim _{n \rightarrow \infty} \frac{\log n}{-\log e_{n, r}}=D_{r}$ is shown in [5], Corollary 11.4 (b). To prove Theorem 3.1 it, therefore, remains to verify that $0<\liminf _{n \rightarrow \infty} n e_{n, r}^{D_{r}}$ for all $r \in[0,+\infty)$. To establish this inequality we will need a series of lemmas.

Lemma 4.1. For every finite set $\alpha \subset \mathbb{R}^{d}$ the function $\mathbb{R}^{d} \rightarrow[-\infty,+\infty]$, $x \rightarrow \log d\left(x, \alpha \cup U^{c}\right)$ is P-integrable. $\left(U^{c}:=\mathbb{R}^{d} \backslash U, \log 0:=-\infty\right)$.

Proof. For every $x \in \mathbb{R}^{d}$ we have

$$
\log d\left(x, \alpha \cup U^{c}\right)=\min \left(\log d(x, \alpha), \log d\left(x, U^{c}\right)\right) .
$$

According to [2], Prop. 3.4 the map $x \mapsto \log d\left(x, U^{c}\right)$ is $P$-integrable. It follows from Proposition $5.1 \mathrm{~b}$ ) and the proof of Lemma 2.6 in [4] that $x \mapsto \log d(x, \alpha)$ is $P$-integrable and the lemma is proved.

Definition 4.2. For every natural number $n \geq 1$ define

$$
u_{n, 0}=\inf \left\{\exp \int \log d\left(x, \alpha \cup U^{c}\right) d P(x) \mid \alpha \subset \mathbb{R}, \operatorname{card}(\alpha) \leq n\right\}, \hat{u}_{n, 0}=\log u_{n, 0},
$$

and, for $0<r<+\infty$,

$$
u_{n, r}=\inf \left\{\int d\left(x, \alpha \cup U^{c}\right)^{r} d P(x) \mid \alpha \subset \mathbb{R}^{d}, \operatorname{card}(\alpha) \leq n\right\} .
$$

Remark 4.3. Obviously we have $u_{n, 0} \leq e_{n, 0}$ and, for $0<r<\infty$; also $u_{n, r} \leq e_{n, r}^{r}$. The main idea in the proof of $0<\liminf _{n \rightarrow \infty} n e_{n, r}^{D_{r}}$ is to replace $e_{n, r}$ by $u_{n, r}^{\frac{1}{r}}$ and then to use the techniques developed in [4] and [5] for the proof of $\liminf _{n \rightarrow \infty} n e_{n, r}^{D_{r}}>0$ in the case of strongly separated self-similar probabilities. 
Lemma 4.4. For every $r \in[0,+\infty)$ and every $n \in \mathbb{N}$ there exists a set $\alpha_{n} \subset \mathbb{R}^{d}$ with $\operatorname{card}\left(\alpha_{n}\right) \leq n$ and

$$
u_{n, r}= \begin{cases}\exp \int \log d\left(x, \alpha_{n} \cup U^{c}\right) d P(x) & \text { if } r=0 \\ \int d\left(x, \alpha_{n} \cup U^{c}\right)^{r} d P(x) & \text { if } r>0 .\end{cases}
$$

Proof. $r=0$ : Let $\bar{U}$ be the closure of $U$ and define $f: \bar{U}^{n} \rightarrow \mathbb{R}$ by

$$
f\left(x_{1}, \ldots, x_{n}\right)=\int \log d\left(x,\left\{x_{1}, \ldots, x_{n}\right\} \cup U^{c}\right) d P(x) .
$$

We will show that $f$ is continuous. Let $\left(x_{1}, \ldots, x_{n}\right) \in \bar{U}^{n}$ be arbitrary and let $\left(\left(x_{1, k}, \ldots, x_{n, k}\right)\right)_{k \in \mathbb{N}}$ be an arbitrary sequence in $\bar{U}^{n}$ with

$$
\lim _{k \rightarrow \infty}\left(x_{1, k}, \ldots, x_{n, k}\right)=\left(x_{1}, \ldots, x_{n}\right) .
$$

Since, for every $x \in A$ and every $\left(y_{1}, \ldots, y_{n}\right) \in \bar{U}^{n}$,

$\log d\left(x,\left\{y_{1}, \ldots, y_{n}\right\} \cup U^{c}\right)=\min \left(\left\{\log \left\|x-y_{i}\right\| \mid i=1, \ldots, n\right\} \cup\left\{\log d\left(x, U^{c}\right)\right\}\right)$ $\leq \log d\left(x, U^{c}\right)$

and since $x \mapsto \log d\left(x, U^{c}\right)$ is $P$-integrable (see [2], Prop. 3.4) we deduce from Lebesgue's dominated convergence theorem that

$$
\begin{aligned}
& \lim _{k \rightarrow \infty} \int \log _{+} d\left(x,\left\{x_{1, k}, \ldots, x_{n, k}\right\} \cup U^{c}\right) d P(x) \\
= & \int \log _{+} d\left(x,\left\{x_{1}, \ldots, x_{n}\right\} \cup U^{c}\right) d P(x) .
\end{aligned}
$$

Since $\int g d P=\int_{0}^{\infty} P(g \geq t) d t$ for every non-negative measurable $g: \mathbb{R}^{d} \rightarrow \mathbb{R}$, by an obvious substitution

$$
\begin{aligned}
& \int \log _{-} d\left(x,\left\{y_{1}, \ldots, y_{n}\right\} \cup U^{c}\right) d P(x) \\
= & \int_{0}^{1} P\left(\left\{x \in A \mid d\left(x,\left\{y_{1}, \ldots, y_{n}\right\} \cup U^{c}\right) \leq s\right\}\right) \frac{d s}{s} .
\end{aligned}
$$

Now we have

$$
\begin{aligned}
& P\left(\left\{x \in A \mid d\left(x,\left\{y_{1}, \ldots, y_{n}\right\} \cup U^{c}\right) \leq s\right\}\right) \\
= & P\left(\left\{x \in A \mid \exists \in 1, \ldots, n:\left\|x-y_{i}\right\| \leq s\right\} \cup\left\{x \in A \mid d\left(x, U^{c}\right) \leq s\right\}\right) \\
\leq & \sum_{i=1}^{n} P\left(B\left(y_{i}, s\right)\right)+P\left(\left\{x \in A \mid d\left(x, U^{c}\right) \leq s\right\}\right)
\end{aligned}
$$


where $B\left(y_{i}, s\right)$ is the closed ball with radius $s$ and center $y_{i}$. Since

$\int_{0}^{1} P\left(\left\{x \in A \mid d\left(x, U^{c}\right) \leq s\right\}\right) \frac{d s}{s}=\int \log _{-} d\left(x, U^{c}\right) d P(x)<+\infty$,

$\int\left(\sup _{y \in \mathbb{R}^{d}} P(B(y, s)) \frac{1}{s}\right) d s<+\infty$ (see [4], Prop. 5.1a) and for $\lambda-$ a.a. $s \in[0,+\infty)$,

$$
\begin{aligned}
& \lim _{k \rightarrow \infty} P\left(\left\{x \in A \mid d\left(x,\left\{x_{1, k}, \ldots, x_{n, k}\right\} \cup U^{c}\right) \leq s\right\}\right) \\
= & P\left(\left\{x \in A \mid d\left(x,\left\{x_{1}, \ldots, x_{n}\right\} \cup U^{c}\right) \leq s\right\}\right) .
\end{aligned}
$$

Lebesgue's dominated convergence theorem implies

$$
\begin{aligned}
& \lim _{k \rightarrow \infty} \int_{0}^{1} P\left(\left\{x \in A \mid d\left(x,\left\{x_{1, k}, \ldots, x_{n, k}\right\} \cup U^{c}\right) \leq s\right\}\right) \frac{d s}{s} \\
= & \int_{0}^{1} P\left(\left\{x \in A \mid d\left(x,\left\{x_{1}, \ldots, x_{n}\right\} \cup U^{c}\right) \leq s\right\}\right) \frac{d s}{s} .
\end{aligned}
$$

Hence

$$
\begin{aligned}
& \lim _{k \rightarrow \infty} \int \log _{-} d\left(x,\left\{x_{1, k}, \ldots, x_{n, k}\right\} \cup U^{c}\right) d P(x) \\
= & \int \log _{-} d\left(x,\left\{x_{1}, \ldots, x_{n}\right\}\right) d P(x) .
\end{aligned}
$$

Combining the preceding results yields the continuity of $f$. Since $\bar{U}^{n}$ is compact, $f$ attains its minimum at some $\left(a_{1}, \ldots, a_{n}\right) \in \bar{U}^{n}$ and $\alpha_{n}=\left\{a_{1}, \ldots, a_{n}\right\}$ satisfies the conclusion of the lemma for $r=0$.

$r>0$ : Define $f: \bar{U}^{n} \rightarrow \mathbb{R}$ by

$$
f\left(x_{1}, \ldots, x_{n}\right)=\int d\left(x,\left\{x_{1}, \ldots, x_{n}\right\} \cup U^{c}\right)^{r} d P(x) .
$$

Using similar techniques as above one can see that $f$ is continuous. Hence it attains its minimum at some point $\left(a_{1}, \ldots, a_{n}\right) \in \bar{U}^{n}$. Obviously this minimum equals $u_{n, r}$ and $\alpha_{n}=\left\{a_{1}, \ldots, a_{n}\right\}$ has the desired property. 


\subsection{Definition and Remark}

For a finite set $\alpha \subset \mathbb{R}^{d}$ and $a \in \alpha$ the set

$$
W(a \mid \alpha)=\left\{x \in \mathbb{R}^{d} \mid\|x-a\|=d(x, \alpha)\right\}
$$

is called the Voronoi cell of $a$ with respect to $\alpha$. A partition $\left(B_{a}\right)_{a \in \alpha}$ of $\mathbb{R}^{d}$ into Borel sets is said to be a Voronoi partition w.r.t. $\alpha$ iff $B_{a} \subset W(a \mid \alpha)$ for all $a \in \alpha$. It is obvious that, for every finite set $\alpha \subset \mathbb{R}^{d}$, there exists a Voronoi partition w.r.t. $\alpha$.

Although an analogous result holds for $r \in(0,+\infty)$ we will need (and formulate) the following lemma only for $r=0$.

Lemma 4.5. For $n \in \mathbb{N}$ let $\alpha_{n} \subset \mathbb{R}^{d}$ satisfy $\operatorname{card}\left(\alpha_{n}\right) \leq n$ and

$$
u_{n, 0}=\exp \int \log d\left(x, \alpha_{n} \cup U^{c}\right) d P(x) \text { (cf. Lemma 4.4). }
$$

Moreover, let $C_{n}=\left\{x \in \mathbb{R}^{d} \mid d\left(x, \alpha_{n}\right) \geq d\left(x, U^{c}\right)\right\}$ and let $\left(B_{a}\right)_{a \in \alpha}$ be a Voronoi partition with respect to $\alpha_{n}$. Let $\gamma_{n}=\left\{a \in \alpha_{n} \mid P\left(B_{a} \backslash C_{n}\right)>0\right\}$. Then $\operatorname{card}\left(\gamma_{n}\right)=n$, in particular $\alpha_{n} \subset U$ and $\operatorname{card}\left(\alpha_{n}\right)=n$.

Proof. First we will show that $\hat{u}_{n, 0}=\int \log d\left(x, \gamma_{n} \cup U^{c}\right) d P(x)$. The inequality $\hat{u}_{n, 0} \leq \int \log d\left(x, \gamma_{n} \cup U^{c}\right) d P(x)$ holds by the definition of $u_{n, 0}$ and $\hat{u}_{n, 0}$. To show the converse inequality note that, for every $a \in \gamma_{n}$ and every $x \in B_{a} \backslash C_{n}$,

$$
d\left(x, \alpha_{n}\right)=\|x-a\| \geq d\left(x, \gamma_{n}\right) \geq d\left(x, \alpha_{n}\right) .
$$

Hence $d\left(x, \alpha_{n}\right)=d\left(x, \gamma_{n}\right)$. Using this fact we obtain

$$
\begin{aligned}
\hat{u}_{n, 0} & =\int \log d\left(x, \alpha_{n} \cup U^{c}\right) d P(x) \\
& =\sum_{a \in \gamma_{n}} \int_{B_{a} \backslash C_{n}} \log d\left(x, \alpha_{n}\right) d P(x)+\int_{C_{n}} \log d\left(x, U^{c}\right) d P(x) \\
& =\sum_{a \in \gamma_{n}} \int_{B_{a} \backslash C_{n}} \log d\left(x, \gamma_{n}\right) d P(x)+\int_{C_{n}} \log d\left(x, U^{c}\right) d P(x) \\
& =\int_{C_{n}^{c}} \log d\left(x, \gamma_{n}\right) d P(x)+\int_{C_{n}} \log d\left(x, U^{c}\right) d P(x) \\
& \geq \int \log d\left(x, \gamma_{n} \cup U^{c}\right) d P(x) .
\end{aligned}
$$


Next, we claim that for every $a \in \gamma_{n}$ there exists a $b \in B_{a}$ such that $P\left(\{x \in A \mid\|x-a\|>\|x-b\|\} \cap\left(B_{a} \backslash C_{n}\right)\right)>0$. Let $a \in \gamma_{n}$ be arbitrary. Since $P\left(B_{a} \backslash C_{n}\right)>0$ and $P$ is continuous we have $P\left(B_{a} \backslash\left(\{a\} \cup C_{n}\right)\right)>0$. Hence, there is a compact set $K \subset B_{a} \backslash\left(\{a\} \cup C_{n}\right)$ with $P(K)>0$. The open sets $U_{b}=\left\{x \in \mathbb{R}^{d} \mid\|x-a\|>\|x-b\|\right\}, b \in K$ form a covering of $K$ since, for every $b \in K$, we have $b \in U_{b}$. Thus, there exists a finite set $\beta \subset K$ with $K \subset \bigcup_{b \in \beta} U_{b}$ which implies $P\left(K \cap U_{b}\right)>0$ for some $b \in \beta \subset K$ and proves our claim.

Finally we prove $\operatorname{card}\left(\gamma_{n}\right)=n$. Assume to the contrary that $\operatorname{card}\left(\gamma_{n}\right)<n$. Choose $a_{0} \in \gamma_{n}$ and $b \in B_{a_{0}}$ with $P\left(U_{b} \cap\left(B_{a_{0}} \backslash C_{n}\right)\right)>0$. Then we get

$$
\begin{aligned}
\hat{u}_{n, 0} \leq & \int \log d\left(x, \gamma_{n} \cup\{b\} \cup U^{c}\right) d P(x) \\
= & \sum_{a \in \gamma_{n} \backslash\left\{a_{0}\right\}} \int_{B_{a} \backslash C_{n}} \log d\left(x, \gamma_{n} \cup\{b\} \cup U^{c}\right) d P(x) \\
& +\int_{B_{a_{o}} \backslash C_{n}} \log d\left(x, \gamma_{n} \cup\{b\} \cup U^{c}\right) d P(x)+\int_{C_{n}} \log d\left(x, \gamma_{n} \cup\{b\} \cup U^{c}\right) d P(x) \\
\leq & \sum_{a \in \gamma \backslash\left\{a_{0}\right\}} \int_{B_{a} \backslash C_{n}} \log d\left(x, \gamma_{n} \cup U^{c}\right) d P(x)+\int_{\left(B_{a_{0}} \backslash C_{n}\right) \cap U_{b}} \log \|x-b\| d P(x) \\
& +\int_{\left(B_{a_{0}} \backslash C_{n}\right) \backslash U_{b}} \log \left\|x-a_{0}\right\| d P(x)+\int_{C_{n}} \log d\left(x, \gamma_{n} \cup U^{c}\right) d P(x) .
\end{aligned}
$$

Since

$$
\int_{\left(B_{a_{0}} \backslash C_{n}\right) \cap U_{b}} \log \|x-b\| d P(x)<\int_{\left(B_{a_{0}} \backslash C_{n}\right) \cap U_{b}} \log \left\|x-a_{0}\right\| d P(x)
$$

and since

$$
\int_{B_{a_{0}} \backslash C_{n}} \log \left\|x-a_{0}\right\| d P(x)=\int_{B_{a_{0}} \backslash C_{n}} \log d\left(x, \gamma_{n}\right) d P(x),
$$

we deduce

$$
\begin{aligned}
\hat{u}_{n, 0} & <\sum_{a \in \gamma_{n}} \int_{B_{a} \backslash C_{n}} \log d\left(x, \gamma_{n}\right) d P(x)+\int_{C_{n}} \log d\left(x, \gamma_{n}\right) d P(x), \\
& \leq \hat{u}_{n, 0},
\end{aligned}
$$

a contradiction. Thus, the lemma is proved. 
Lemma 4.6. $\lim _{n \rightarrow \infty}\left(\hat{u}_{n, 0}-\hat{u}_{n+1,0}\right)=0$.

Proof. Let $\alpha_{n+1} \subset \mathbb{R}^{d}$ satisfy $\operatorname{card}\left(\alpha_{n+1}\right) \leq n+1$ and

$$
\hat{u}_{n+1,0}=\int \log d\left(x, \alpha_{n+1} \cup U^{c}\right) d P(x) .
$$

According to Lemma 4.6 we have $\operatorname{card}\left(\alpha_{n+1} \cap U\right)=n+1$. Let $\left(B_{a}\right)_{a \in \alpha_{n+1}}$ and $C_{n+1}$ be as in Lemma 4.6. Then there exists an $a_{0} \in \alpha_{n+1}$ with $P\left(B_{a_{0}}\right) \leq \frac{1}{n+1}$, and we get

$$
\begin{aligned}
\hat{u}_{n, 0} \leq & \int \log d\left(x,\left(\alpha_{n+1} \backslash\left\{a_{0}\right\}\right) \cup U^{c}\right) d P(x) \\
\leq & \sum_{a \in \alpha_{n+1} \backslash\left\{a_{0}\right\}} \int_{B_{a} \backslash C_{n+1}} \log d\left(x,\left(\alpha_{n+1} \backslash\left\{a_{0}\right\}\right) \cup U^{c}\right) d P(x) \\
& +\int_{B_{a_{0} \backslash C_{n+1}}} \log d\left(x, U^{c}\right) d P(x)+\int_{C_{n+1}} \log d\left(x, U^{c}\right) d P(x) \\
= & \sum_{a \in \alpha_{n+1}} \int_{B_{a} \backslash C_{n+1}} \log \|x-a\| d P(x)-\int_{B_{a_{0}} \backslash C_{n+1}} \log \left\|x-a_{0}\right\| d P(x) \\
& +\int_{B_{a_{0}} \backslash C_{n+1}} \log d\left(x, U^{c}\right) d P(x)+\int_{C_{n+1}} \log d\left(x, U^{c}\right) d P(x) .
\end{aligned}
$$

Since $A$ is bounded, there exists $a c \in(1,+\infty)$ with $\log d\left(x, U^{c}\right) \leq c$ for all $x \in A$. For every $x \in C_{n+1}$ we have $d\left(x, U^{c}\right)=d\left(x, \alpha_{n+1} \cup U^{c}\right)$ and

$$
\sum_{a \in \alpha_{n+1}} \int_{B_{a} \backslash C_{n+1}} \log \|x-a\| d P(x)=\int_{C_{n+1}^{c}} \log d\left(x, \alpha_{n+1} \cup U^{c}\right) d P(x) .
$$

Thus, we deduce

$$
\hat{u}_{n, 0} \leq \hat{u}_{n+1,0}-\int_{B_{a_{0}} \backslash C_{n+1}} \log \left\|x-a_{0}\right\| d P(x)+c P\left(B_{a_{0}} \backslash C_{n+1}\right) .
$$


Now

$$
\begin{aligned}
\int_{B_{a_{0} \backslash C_{n+1}} \log \left\|x-a_{0}\right\| d P(x)} & \int_{\left(B_{a_{0} \backslash C_{n+1}}\right) \cap \bar{B}\left(a_{0}, 1\right)} \log \left\|x-a_{0}\right\| d P(x) \\
& =-\int_{0}^{1} P\left(\left(B_{a_{0}} \backslash C_{n+1}\right) \cap B\left(a_{0}, s\right)\right) \frac{d s}{s} .
\end{aligned}
$$

Let $p>1$ and $q$ with $\frac{1}{p}+\frac{1}{q}=1$. Then Hölder's inequality yields

$$
P\left(\left(B_{a_{0}} \backslash C_{n+1}\right) \cap B\left(a_{0}, s\right)\right) \leq P\left(B_{a_{0}} \backslash C_{n+1}\right)^{\frac{1}{p}} P\left(B\left(a_{0}, s\right)\right)^{\frac{1}{q}} .
$$

By [4], Prop. 5.1 a), there is a $C \in \mathbb{R}$ and $t>0$ with, $P(B(x, s)) \leq C s^{t}$ for all $x \in \mathbb{R}^{d}$ and all $s \in[0,1]$. Hence we obtain

$$
\begin{aligned}
\hat{u}_{n, 0} & \leq \hat{u}_{n+1,0}+\int_{0}^{1} P\left(\left(B_{a_{0}} \backslash C_{n+1}\right)\right)^{\frac{1}{p}} C s^{t} \frac{d s}{s}+c P\left(B_{a_{0}} \backslash C_{n+1}\right) \\
& \leq \hat{u}_{n+1,0}+\left(\frac{1}{n+1}\right)^{\frac{1}{p}} c \frac{1}{t}+\frac{1}{n+1} c .
\end{aligned}
$$

Hence, the lemma is proved.

Lemma 4.7. Let $r \in[0,+\infty)$ and, for each $n \in \mathbb{N}$, let the set $\alpha_{n} \subset \mathbb{R}^{d}$ satisfy $\operatorname{card}\left(\alpha_{n}\right) \leq n$ and

$$
u_{n, r}= \begin{cases}\exp \int \log d\left(x, \alpha_{n} \cup U^{c}\right) d P(x) & \text { if } r=0 \\ \int d\left(x, \alpha_{n} \cup U^{c}\right)^{r} d P(x) & \text { if } r>0 .\end{cases}
$$

Set $\delta_{n}=\max _{x \in A} d\left(x, \alpha_{n} \cup U^{c}\right)$. Then $\lim _{n \rightarrow \infty} \delta_{n}=0$.

Proof. Since $P$ is continuous and $P(A \cap U)=1$ (follows from [2], Proposition $3.4)$, we have $\delta_{n}>0$ for all $n \in \mathbb{N}$. For each $n \in \mathbb{N}$ there is an $x_{n} \in A$ and an $a_{n} \in \alpha_{n} \cup U^{c}$ with $\left\|x_{n}-a_{n}\right\|=d\left(x_{n}, \alpha_{n} \cup U^{c}\right)=\delta_{n}$. For all $x \in A \cap B\left(x_{n}, \frac{1}{2} \delta_{n}\right)$ and all $a \in \alpha_{n} \cup U^{c}$ we have

$$
\|x-a\| \geq\left\|x_{n}-a\right\|-\left\|x_{n}-x\right\| \geq\left\|x_{n}-a_{n}\right\|-\frac{1}{2} \delta_{n}=\frac{1}{2} \delta_{n} .
$$

$r=0$ : Set $\beta_{n+1}=\alpha_{n} \cup\left\{x_{n}\right\}$. Then $x \in A \cap B\left(x_{n}, \frac{1}{2} \delta_{n}\right)$ implies

$$
d\left(x, \beta_{n+1} \cup U^{c}\right)=\left\|x-x_{n}\right\| .
$$


Thus we deduce

$$
\begin{aligned}
\hat{u}_{n+1,0} \leq & \int \log d\left(x, \beta_{n+1} \cup U^{c}\right) d P(x) \\
& \leq \int_{B\left(x_{n}, \frac{\delta_{n}}{2}\right)} \log \left\|x-x_{n}\right\| d P(x)+\int_{A \backslash B\left(x_{n}, \frac{\delta_{n}}{2}\right)} \log d\left(x, \alpha_{n} \cup U^{c}\right) d P(x) \\
& =\int \log d\left(x, \alpha_{n} \cup U^{c}\right) d P(x)-\int_{B\left(x_{n}, \frac{\delta_{n}}{2}\right)} \log d\left(x, \alpha_{n} \cup U^{c}\right) d P(x) \\
& +\int_{B\left(x_{n}, \frac{\delta_{n}}{2}\right)} \log \left\|x-x_{n}\right\| d P(x) .
\end{aligned}
$$

Since $d\left(x, \alpha_{n} \cup U^{c}\right) \geq \frac{\delta_{n}}{2}$ for all $x \in A \cap B\left(x_{n}, \frac{\delta_{n}}{2}\right)$, we obtain

$$
\int_{B\left(x_{n}, \frac{\delta_{n}}{2}\right)} \log d\left(x, \alpha_{n} \cup U^{c}\right) d P(x) \geq P\left(B\left(x_{n} \frac{\delta_{n}}{2}\right)\right) \log \frac{\delta_{n}}{2}
$$

and therefore,

$$
\hat{u}_{n, 0}-\hat{u}_{n+1,0} \geq P\left(B\left(x_{n}, \frac{\delta_{n}}{2}\right)\right) \log \frac{\delta_{n}}{2}-\int_{B\left(x_{n}, \frac{\delta_{n}}{2}\right)} \log \left\|x-x_{n}\right\| d P(x) .
$$

If $\delta_{n} \leq 2$, then it follows that $\hat{u}_{n, 0}-\hat{u}_{n+1,0} \geq \int_{0}^{\frac{\delta_{n}}{2}} P\left(B\left(x_{n}, s\right)\right) \frac{d s}{s}$. If $\delta_{n}>2$, then it follows that

$$
\begin{aligned}
\hat{u}_{n, 0}-\hat{u}_{n+1,0} \geq & P\left(B\left(x_{n}, \frac{\delta_{n}}{2}\right)\right) \log \frac{\delta_{n}}{2}-\int_{B\left(x_{n}, \frac{\delta_{n}}{2}\right) \backslash B\left(x_{n}, 1\right)} \log \frac{\delta_{n}}{2} d P(x) \\
& -\int_{B\left(x_{n}, 1\right)} \log \left\|x-x_{n}\right\| d P(x) \geq \int_{0}^{1} P\left(B\left(x_{n}, s\right)\right) \frac{d s}{s} .
\end{aligned}
$$

Since, for every $w \in[0,1]$, the map $x \mapsto \int_{0}^{w} P(B(x, s)) \frac{d s}{s}$ is continuous, we have $g(w)=\min _{x \in A} \int_{0}^{w} P(B(x, s)) \frac{d s}{s}>0$ for $w>0$ and the function $g:[0,1] \rightarrow \mathbb{R}$ is nondecreasing. We obtain $\hat{u}_{n, 0}-\hat{u}_{n+1,0} \geq g\left(\min \left(1, \frac{\delta_{n}}{2}\right)\right)$. Now because $\lim _{n \rightarrow \infty}\left(\hat{u}_{n, 0}-\hat{u}_{n+1,0}\right)=0$ (see Lemma 4.7) this implies $\lim _{n \rightarrow \infty} \delta_{n}=0$. 
$r>0:$ From $(*)$ we deduce

$$
\begin{aligned}
u_{n, r} & =\int d\left(x, \alpha_{n} \cup U^{c}\right)^{r} d P(x) \\
& \geq \int_{B\left(x_{n}, \frac{\delta_{n}}{2}\right)}\left(\frac{1}{2} \delta_{n}\right)^{r} d P(x)=\left(\frac{1}{2} \delta_{n}\right)^{r} P\left(B\left(x_{n}, \frac{1}{2} \delta_{n}\right)\right) .
\end{aligned}
$$

Assume $\lim \sup \delta_{n}>\delta>0$. Then $\delta_{n}>\delta$ for infinitely many $n$ and hence $\left.u_{n, r} \geq P\left(\stackrel{n \rightarrow \infty}{B\left(x_{n}\right.}, \frac{1}{2} \delta\right)\right)\left(\frac{1}{2} \delta\right)^{r}$ for infinitely many $n$. Since $\min _{x \in A} P\left(B\left(x, \frac{1}{2} \delta\right)\right)>0$ this implies $\limsup _{n \rightarrow \infty} u_{n, r}>0$. Since $e_{n, r}^{r} \geq u_{n, r}$ and $\lim _{n \rightarrow \infty} e_{n, r}=0$ (see [5], Lemma 6.1). This yields a contradiction and the lemma is proved.

Lemma 4.8. Let $r \in[0,+\infty)$ be given. Then there exists an $n_{0} \in \mathbb{N}$ such that, for all $n \geq n_{0}$, there are $n_{1}(n), \ldots, n_{N}(n) \in \mathbb{N}$ with $n_{i}(n) \geq 1, \sum_{i=1}^{N} n_{i}(n) \leq n$, and

$$
u_{n, r} \geq \begin{cases}\prod_{i=1}^{N}\left(s_{i} u_{n_{i}(n), 0}\right)^{p_{i}} & \text { if } r=0 \\ \sum_{i=1}^{N} p_{i} s_{i}^{r} u_{n_{i}(n), r} & \text { if } r>0 .\end{cases}
$$

Proof. There is a $\tau \in\{1, \ldots, \mathbb{N}\}^{*}$ with $A_{\tau} \subset U$ (see, for instance, [2], proof of Lemma 3.3). Then $\varepsilon=d\left(A_{\tau}, U^{c}\right)>0$. Set $s_{\min }=\min \left\{s_{1}, \ldots, s_{N}\right\}$. We deduce $d\left(S_{i}\left(A_{\tau}\right), S_{i}(U)^{c}\right)=s_{i} d\left(A_{\tau}, U^{c}\right) \geq s_{\min } \varepsilon$ and, hence, that $d\left(x, U^{c}\right) \geq$ $d\left(x, S_{i}(U)^{c}\right) \geq s_{\min } \varepsilon$ for all $x \in S_{i}\left(A_{\tau}\right)$. Let $\alpha_{n}$ and $\delta_{n}$ be as in Lemma 4.8 and choose $n_{0}$ such that $\delta_{n}<s_{\min } \varepsilon$ for all $n \geq n_{0}$. Let $n \geq n_{0}$ and $x \in S_{i}\left(A_{\tau}\right)$ be fixed for the moment. Then there exists an $a \in \alpha_{n} \cup U^{c}$ with $\|x-a\|=d\left(x, \alpha_{n} \cup U^{c}\right) \leq \delta_{n}<s_{\min } \varepsilon$. Thus we get $a \in S_{i}(U) \subset U$ and, therefore, $S_{i}(U) \cap \alpha_{n} \neq \emptyset$.

Now define $\alpha_{n, i}=\alpha_{n} \cap S_{i}(U)$ and $n_{i}=\operatorname{card}\left(\alpha_{n, i}\right)$. Then $n_{i} \geq 1$ and, since $S_{i}(U) \cap S_{j}(U)=\emptyset$ for $i \neq j, \sum_{i=1}^{N} n_{i} \leq \operatorname{card}\left(\alpha_{n}\right) \leq n$.

Using the self-similarity of $P$ and the fact that $S_{i}(U) \subset U$, we obtain for $r=0$

$$
\begin{aligned}
\hat{u}_{n, 0} & =\sum_{i=1}^{N} p_{i} \int \log d\left(S_{i} x, \alpha_{n} \cup U^{c}\right) d P(x) \\
& \geq \sum_{i=1}^{N} p_{i} \int \log d\left(S_{i} x, \alpha_{n} \cup S_{i}(U)^{c}\right) d P(x)
\end{aligned}
$$




$$
\begin{aligned}
& =\sum_{i=1}^{N} p_{i} \int \log d\left(S_{i} x, \alpha_{n, i} \cup S_{i}(U)^{c}\right) d P(x) \\
& =\sum_{i=1}^{N} p_{i} \int \log \left(s_{i} d\left(x, S_{i}^{-1}\left(\alpha_{n, i}\right) \cup U^{c}\right) d P(x)\right. \\
& =\sum_{i=1}^{N} p_{i} \log s_{i}+\sum_{i=1}^{N} p_{i} \int \log d\left(x, S_{i}^{-1}\left(\alpha_{n, i}\right) \cup U^{c}\right) d P(x) \\
& \geq \sum_{i=1}^{N} p_{i} \log s_{i}+\sum_{i=1}^{N} p_{i} \hat{u}_{n_{i} 0}
\end{aligned}
$$

and, for $r>0$,

$$
\begin{aligned}
u_{n, r} & =\sum_{i=1}^{N} p_{i} \int d\left(S_{i} x, \alpha_{n} \cup U^{c}\right)^{r} d P(x) \\
& \geq \sum_{i=1}^{N} p_{i} s_{i}^{r} \int d\left(x, S_{i}^{-1}\left(\alpha_{n, i}\right) \cup U^{c}\right)^{r} d P(x) \geq \sum_{i=1}^{N} p_{i} s_{i}^{r} u_{n_{i}, r} .
\end{aligned}
$$

Thus the lemma is proved.

\section{Lemma 4.9.}

a) $\inf \left\{n^{\frac{1}{D_{0}}} u_{n, 0}: n \in \mathbb{N}\right\}>0$

b) $\inf \left\{n^{\frac{r}{D_{r}}} u_{n, r}: n \in \mathbb{N}\right\}>0$ for $r \in(0,+\infty)$.

\section{Proof.}

a) It is enough to show $\inf \left\{\frac{1}{D_{0}} \log n+\hat{u}_{n, 0}: n \in \mathbb{N}\right\}>-\infty$. It follows from Lemma 4.1 and Lemma 4.4 that $\hat{u}_{n, 0}>-\infty$ for all $n \in \mathbb{N}$. Let $n_{0} \in \mathbb{N}$ and, for $n \geq n_{0}, n_{1}(n), \ldots, n_{N}(n)$ be as in Lemma 4.9. Set

$$
c=\min \left\{\frac{1}{D_{0}} \log n+\hat{u}_{n, 0}: n \leq n_{0}\right\} .
$$


If $n \geq n_{0}$ and $\frac{1}{D_{0}} \log k+\hat{u}_{k, 0} \geq c$ for all $k \leq n-1$, then

$$
\begin{aligned}
\hat{u}_{n .0} & \geq \sum_{i=1}^{N} p_{i} \log s_{i}+\sum_{i=1}^{N} p_{i} \hat{u}_{n_{i}(n), 0} \\
& \geq \sum_{i=1}^{N} p_{i} \log s_{i}+\sum_{i=1}^{N} p_{i}\left(c-\frac{1}{D_{0}} \log n_{i}(n)\right) \\
& \geq c-\frac{1}{D_{0}} \log n+\sum_{i=1}^{N} p_{i} \log s_{i}-\frac{1}{D_{0}} \sum_{i=1}^{N} p_{i} \log \frac{n_{i}(n)}{n} .
\end{aligned}
$$

Since $\sum_{i=1}^{N} p_{i} \log \frac{n_{i}(n)}{n} \leq \sum_{i=1}^{N} p_{i} \log p_{i}$, we get

$$
\frac{1}{D_{0}} \log n+\hat{u}_{n, 0} \geq c+\sum_{i=1}^{N} p_{i} \log s_{i}-\frac{1}{D_{0}} \sum_{i=1}^{N} p_{i} \log p_{i}=c .
$$

By induction we obtain $\inf \left\{\frac{1}{D_{0}} \log n+\hat{u}_{n, 0} \mid n \in \mathbb{N}\right\} \geq c>-\infty$.

b) Let $\alpha_{n}$ be as in Lemma 4.8. Since $P\left(A \cap\left(\alpha_{n} \cup U^{c}\right)\right)=0$ and $d\left(x, \alpha_{n} \cup U^{c}\right)>0$ for all $x \in A \backslash\left(\alpha_{n} \cup U^{c}\right)$ we get $u_{n, r}>0$ for all $n \in \mathbb{N}$. Let $n_{0}$ and, for $n \geq n_{0}$, $n_{1}(n), \ldots, n_{N}(n)$ be as in Lemma 4.9. Set $c=\min \left\{n \frac{r}{D_{r}} u_{n, r}: n \leq n_{0}\right\}$. Then we have $c>0$. Let $n \geq n_{0}$ be such that $k^{\frac{r}{D_{r}}} u_{k, r} \geq c$ for all $k \leq n-1$. Using Lemma 4.9 we deduce

$$
n^{\frac{r}{D_{r}}} u_{n, r} \geq n^{\frac{r}{D_{r}}} \sum_{i=1}^{N} p_{i} s_{i}^{r} n_{i}(n)^{-\frac{r}{D_{r}}} n_{i}(n)^{\frac{r}{D_{r}}} u_{n_{i}(n), r}
$$

Since $n_{i}(n)<n$, we obtain $n^{\frac{r}{D_{r}}} u_{n, r} \geq c \sum_{i=1}^{N} p_{i} s_{i}^{r}\left(\frac{n_{i}(n)}{n}\right)^{-\frac{r}{D_{r}}}$. Using Hölder's inequality (exponents less than 1 ) yields

$$
\sum_{i=1}^{N} p_{i} s_{i}^{r}\left(\frac{n_{i}(n)}{n}\right)^{-\frac{r}{D_{r}}} \geq \sum_{i=1}^{N}\left(p_{i} s_{i}^{r}\right)^{\left(\frac{D_{r}}{r+D_{r}}\right)^{1+\frac{r}{d_{r}}}}\left(\sum_{i=1}^{N}\left(\frac{n_{i}(n)}{n}\right)^{\left(-\frac{r}{D_{r}}\right) \cdot\left(-\frac{D_{r}}{r}\right)}\right)^{-\frac{r}{D_{r}}}=1 .
$$

By induction we get $n^{\frac{r}{D_{r}}} u_{n, r} \geq c$ for all $n \in \mathbb{N}$ and the lemma is proved.

Proof of Theorem 3.1. According to the considerations at the beginning of this section the theorem is proved if one can establish that for all $r \in[0,+\infty)$ 
$0<\liminf _{n \rightarrow \infty} n e_{n, r}^{D_{r}}$. We know that

$$
u_{n, r} \leq \begin{cases}e_{n, r} & \text { if } r=0 \\ e_{n, r}^{r} & \text { if } r>0\end{cases}
$$

and Lemma 4.10 immediately implies $\liminf _{n \rightarrow \infty} n u_{n, 0}^{D_{0}}>0$ and $\lim _{n \rightarrow \infty} \inf n u_{n, r}^{\frac{D_{r}}{r}}>0$ for $r \in(0,+\infty)$. Thus the theorem is proved.

\section{References}

[1] K. Falconer, Techniques in fractal geometry, John Wiley\&Sons, 1997.

[2] S. Graf, On Bandt's tangential distribution for self-similar measures, Mh. Math. 120 (1995), 223-246.

[3] S. Graf and H. Luschgy, The quantization of the Cantor distribution, Math. Nachr. 183 (1997), 113-133.

[4] S. Graf and H. Luschgy, Quantization for probability measures with respect to the geometric mean error, Preprint 1999.

[5] S. Graf and H. Luschgy, Foundations of quantization for probability distributions, Lect. Notes Math. 1730, Springer 2000.

[6] S. Graf and H. Luschgy, The quantization dimension of self-similar probabilities, Math. Nachr., to appear.

[7] A. Schief, Separation properties of self-similar sets, Proc. Amer. Math. Soc. 122 (1994), 111-116. 